\title{
Resultados atuais da cirurgia coronária em pacientes de alto risco
}

\author{
Pedro R. SALERNO*, Kassem A. ASCHE*, Robert J. CUSIMANO**, Samuel V. LICHTENSTEIN**, Tomas \\ A. SALERNO**
}

RBCCV $44205-48$
SALERNO, P. R.; ASCHE, K. A.; CUSIMANO, R J.; LICHTENSTEIN, S. V.; SALERNO, T. A. - Resultados atuais da cirurgia coronária em pacientes de alto risco. Rev. Bras. Cir. Cardiovasc., 3(1): 50-54, 1988.

RESUMO: No periodo e 4 anos (1984 a 1987), 427 pacientes ( 313 homens e 114 mulheres, idade média 61,83 anos) foram submetidos a cirurgia coronária de emergência. $A$ indicação para cirurgia foi angina instável, que requeria uso intravenoso de nitroglicerina. Quanto à classe funcional, $369(86,41 \%)$ pacientes estavam em classe IV e $51,95 \%$ estavam em grau funcional III ou IV de ventrículo esquerdo, pelo estudo hemodinâmico e $30,44 \%$ tinham lesão de tronco. Foram realizadas 2,99 pontes/pacientes. A mortalidade total foi de $7,72 \%$ e $17,09 \%$ tiveram síndrome de baixo débito; déficit neurológico ocorreu em $2,85 \%$ e infarto do miocárdio, em $16,86 \%$ dos casos. Durante o mesmo período, 30 pacientes ( 16 do sexo masculino e 14 do feminino) com idade média de 64,03 anos, com amgina instável e em choque cardiogênico, foram submetidos a revascularizaçāo de emergência. Quanto à classe funcional, $86,66 \%$ estavam em classe IV, $13,33 \%$ em classe III e $56,66 \%$ estavam em grau funcional IV de ventrículo esquerdo e $13,33 \%$ em grau III; $40 \%$ dos pacientes tinham lesão de tronco. O número médio de pontes foi de 2,76 pontes/pacientes. A mortalidade foi de $60 \%$; déficit neurológico ocorreu em $16,66 \%$ e $43,33 \%$ sofreram infarto do miocárdio. Em conclusão, a mortalidade e a morbidade, para pacientes submetidos a cirurgia de revascularização, permanecem elevadas, principalmente, em pacientes com choque cardiogênico. Portanto, mais estudos futuros são requeridos em relaçăo à proteção miocárdica, para melhores resultados. rurgia.

DESCRITORES: miocárdio, revascularizaçâo, pacientes de alto risco; miocárdio, revascularização, ci-

\section{INTRODUÇÃO}

A indicação cirúrgica para pacientes coronarianos vem sofrendo uma mudança, no sentido de se operarem pacientes em grau funcional e fração de ejeção de ventrículo esquerdo com maior deterioração do que no passado.

Hoje, o paciente em choque cardiogênico passou a ter espaço dentro das indicaçōes cirúrgicas para revascularização miocárdica ${ }^{6}$, porém com uma alta taxa de mortalidade pré-operatória ${ }^{5,6,8}$.
Neste estudo, analisamos os resultados de pacientes com angina instável refratária ao tratamento clínico e pacientes em choque cardiogênico que foram submetidos a revascularização do miocárdio, na Universidade de Toronto.

\section{MATERIAL E MÉTODO}

No período de 1984 a 1987, identificamos 427 pacientes que preencheram todos os critérios para angina

Trabalho realizado na University of Toronto. Ontário, Canadá

Apresentado ao 15: Congresso Nacional de Cirurgia Cardiaca. Rio de Janeiro, 7 e 8 de abril, 1988.

- Do Instituto Dante Pazzanese de Cardiologia. São Paulo, SP, Brasil, Fellow da Universidade do Toronto, quando da realizaçăo deste trabalho.

*. Da University of Toronto. Ontário, Canadá.

Endereço para separatas: Pedro R. Salerno. Caixa Postal 215. São Paulo,.SP. Brasil. 
SALERNO, P. R.; ASCHE, K. A.; CUSIMANO, R. J.; LICHTENSTEIN, S. V.; SALERNO, T. A. - Resultados atuais da cirurgia coronária em pacientes de alto risco. Rev. Bras. Cir. Cardiovasc., 3(1): 50-54, 1988.

instável. Estes critérios são: pacientes no centro de terapia intensiva com dor contínua, apesar da máxima terapia médica (nitroglicerina IV $300 \boldsymbol{\mu g}$ /minuto) e balão intraaórtico. No mesmo período, 30 pacientes foram para cirurgia em choque cardiogênico. Os critérios utilizados para caracterização de choque cardiogênico foram: pressão sistólica menor ou igual a $80 \mathrm{mmHg}$, índice cardiaco menor ou igual a $21 / \mathrm{minuto} / \mathrm{m}^{2}$; débito urinário menor ou igual a $20 \mathrm{ml} /$ minuto, pele úmida e fria.

No grupo de angina instável os dados pré-operatórios foram: o sexo masculino esteve presente em $73,3 \%$ (313); a idade média foi de 61,83 anos e lesão de tronco ocorreu em $30.44 \%$ (130) dos casos. Quanto à classe funcional, $86,41 \%(369)$ estavam em classe IV da NYHA; $10,53 \%$ (45), $1,63 \%$ (7) e 1,40\% (6) em classe III, II e I, respectivamente. Consideramos a função ventricular pelo estudo hemodinâmico como menor ou igual a $20 \%$ grau IV; $20-40 \%$ grau III; $40-60 \%$ grau II e maior ou igual a $60 \%$ grau I; portanto, $17,33 \%$ (74) estavam em grau IV; 34,66\% (148), 43,09\% (184), $4,91 \%$ (21) em grau III, II e I, respectivamente. Para estes pacientes, foi realizada uma média de 2,99 pontes/ paciente. Isto requereu uma média de 48 minutos de pinçamento total aórtico e 71 minutos em perfusão. As cirurgias foram realizados utilizando-se perfusão sistêmica hipotérmica a $33^{\circ} \mathrm{C}$, cardioplegia sangüínea contínua e pinçamento único de aorta para a feitura das anastomoses distais e proximais. O sistema utilizado para administração da cardioplegia sangüínea contínua foi o "Buckberg-Shiley Cardioplegia Delivery System""2. Através de um adaptador tipo $\mathrm{Y}$ conectado na aorta ascendente e por 3 vias conectadas diretamente às veias safenas, a cardioplegia é administrada à medida em que as anastomoses distais vão sendo realizadas. Foram utilizados 2 tipos de soluções cardioplégcias, uma com alto teor de potássio, $27 \mathrm{mEq} / \mathrm{l}$, administrado em uma infusão média inicial de $1.000 \mathrm{ml}$ à temperatura de $18^{\circ} \mathrm{C}$ a $20^{\circ} \mathrm{C}$. A seguir, uma solução com baixo teor de potássio, $7 \mathrm{mEq} / \mathrm{l}$ em infusão contínua a $100 \mathrm{ml} /$ minuto. $\mathrm{O}$ hematócrito da solução de cardioplegia foi de $14 \%$ e a pressão parcial de oxigênio, de $237 \mathrm{mmHg}$. O potássio sérico, no pós-operatório, foi de $3,8 \mathrm{mEq} /$ l.

Os pacientes em choque cardiogênico estavam em faixa etária um pouco mais elevada, com idade média de 64 anos e sendo $53,33 \%$ (16) do sexo masculino. Lesão de tronco esteve presente em $40 \%$ (12) dos casos. Quanto à classe funcional, $86,66 \%$ (26) estavam em classe IV da NYHA e $13,33 \%$ (4), em classe III: Em relação à função ventricular pré-choque cardiogênico, $63,33 \%$ (19) estavam em grau IV, $13,33 \%$ (4) em grau III, $16,66 \%$ (2) em grau II e $6,66 \%$ (1) em grau I. Destes pacientes, $26,66 \%$ (8) estiveram em vigência de angina instável, $33,33 \%$ (10) em insuficiência coronária aguda, $23,33 \%$ (7) em infarto subendocárdico e $16,66 \%$ (5) em infarto transmural e evoluíram para choque cardiogênico. Este quadro de choque cardiogênico não reverteu, apesar da terapêutica médica máxima, tal como, nitrogli- cerina IV (300 $\boldsymbol{\mu g} /$ minuto), agentes inotrópicos e balão intra-aórtico. Os dados operatórios, neste grupo, são: 2,75 pontes/paciente, o que requereu um tempo médio de pinçamento da aorta de 67 minutos. Deve-se lembrar que este é um pinçamento único para a feitura das anastomoses distais e proximais e um tempo médio de perfusão de 139 minutos; a proteção miocárdica foi semeIhante à utilizada no grupo de angina instável.

\section{RESULTADOS}

No grupo de angina instável, a mortalidade total hospitalar foi de $7,72 \%$ (33); síndrome de baixo débito ocorreu em $17,09 \%$ (73) dos pacientes e balāo intraaórtico foi utilizado em 17,09\% (73) dos casos. Evidência eletrocardiográfica e enzimática de infarto do miocárdio, no pós-operatório, foi de $16,86 \%$ dos casos e $2,8 \%$ tiveram déficit neurológico por mais de 72 horas, mas que se reverteu antes da alta hospitalar. Nos casos de choque cardiogênico, a mortalidade, em 30 dias, foi de $60 \%$ (18), a síndrome de baixo débito cardíaco esteve presente em $86,66 \%$ (26) e o balāo intra-aórtico foi utilizado em $86,66 \%$ (26) dos casos. Evidência eletrocardiográfica e enzimática de infarto do miocárdio foi de $43,33 \%$, algum grau de déficit neurológico esteve presente em $16,66 \%$ (5), por mais de 72 horas.

\section{DISCUSSÃO}

A análise do resultado cirúrgico, para os pacientes com angina instável e choque cardiogênico, mostra ser este um grupo de pacientes com severos sintomas clínicos e de grande gravidade. Na literatura, os pacientes com angina instável são, também, referidos como insuficiência coronária aguda, angina pós-infarto, infarto do miocárdio em evolução. Portanto, identificar esses pacientes com esta forma de angina é, provavelmente, classificá-los com uma forma mais maligna que a angina pectoris per si. A mortalidade, para estes pacientes, tem sido relatada ser tão alta quanto $36 \% 2,3,4$. Um consenso da literautra é muito difícil, devido à heterogeneidade das definiçōes para angina instável.

Choque cardiogênico é uma síndrome bastante conhecida. Muitos investigadores identificam esses pacientes como de alto risco, tanto com ou sem cirurgia. KILLIP \& $\mathrm{KIMBOLL}^{5}$, em seu trabalho clássico, reportaram mortalidade de $81 \%$ em pacientes tratados clinicamente. Não houve mudanças, nestes resultados, apesar do advento de melhores cuidados em terapia intensiva ${ }^{7}$. Subramanian, em 1980 , relatou mortalidade de $45 \%$, no grupo de pacientes com choque cardiogênico tratados cirurgi- 
SALERNO, P. R.; ASCHE, K. A.; CUSIMANO, R. J.; LICHTENSTEIN, S. V.; SALERNO, T. A. - Resultados atuais da cirurgia coronária em pacientes de alto risco. Rev. Bras. Cir. Cardiovasc., 3(1): 50-54, 1988.

camente. Porém, mais recentemente, ATHANASULEAS et alii ${ }^{1}$ reportam mortalidade hospitalar de $61 \%$.

Neste nosso estudo, encontamos mortalidade hospitalar, para angina instável, de $7,72 \%$ (33) e para choque cardiogênico, de $60 \%$. Quando comparamos estes resultados com pacientes que estiveram em condições instáveis de angina instável e que foram submetidos a cirurgia, a mortalidade em 30 dias foi de $2,2 \%$. Isto reflete, mais uma vez, a gravidade destes pacientes em condições instáveis de angina instável e em choque cardiogênico.

\section{CONCLUSÃO}

Podemos concluir que os resultados cirúrgicos para os pacientes em angina instável e choque cardiogênico mostraram mortalidade elevada, quando comparada com pacientes estáveis. Assim, como a utilização de suporte circulatório foi maior para esses pacientes, a incidência de infarto do miocádio foi, também, mais elevada do que nos casos eletivos. Isto mostra que esses pacientes são portadores de uma baixa reserva miocárdica, o que nos faz refetir que mais estudos futuros são requeridos, em relaçāo à proteçāo miocárdica, para esses pacientes.

RBCCV $44205-48$

SALERNO, P. R.; ASCHE, K. A.; CUSIMANO, R J.; LICHTENSTEIN, S. V.; SALERNO, T. A. - Currente results for coronary artery bypass for high risk patients. Rev. Bras. Cir. Cardiovasc., 3(1): 50-54, 1988.

ABSTRACT: In a 4 year period (1984-1987) 427 patients ( 313 males and 114 females, mean age 61.83 years) underwent emergency aorto-coronary bypass procedures. Indications for surgery were unstable angina, requiring intravenous nitroglycerine. 369 patients $(86.41 \%)$ were class IV NYHA classification and $51.99 \%$ were grade III or IV ventricles. $30.44 \%$ had main left coronary stenosis. Mean number of grafts was $2.99 \%$. Overall mortality was $7.72 \%$ and $17.09 \%$ had low output state. Stroke occured in $2.81 \%$ and the infarction rate was $16.86 \%$. During the same period 30 patients ( 16 males and 14 females mean age 64.03 years) with unstable angina and in cardiogenic shock underwent emergency bypass. $86.66 \%$ were class IV and $13.33 \%$ class III. L. V. grade was IV $56.66 \%$ and III $13.33 \%$. $40 \%$ had main left stenosis. Mean number of grafts was 2.75 . $30 \%$ died in the OR and $30 \%$ died in 30 days. Stroke occured in $16.66 \%$ and $43.33 \%$ suffered a myocardial infartion. In conclusion the mortality and morbidity for patients undergoing emergency coronary artery bypass remain very high. This is particulary so for patients in cardiogenic shock Future developments in cardiplegic method may improve results.

DESCRIPTORS: myocardial revascularization, high risk patients; myocardial revascularization, surgery.

\section{REFERÊNCIAS BIBLIOGRÁFICAS}

1 ATHANASUlEAS, C. L.; GEER, D. A.; ARCINIEGAS, J. G.; COOPER, T. B.; HESS, R. G.; MaCLEAN, W. A. H.; PAPAPIETRO, S. E.; STANLEY, A. W. H.; McEACHERN, M. - A reappraisal of surgical intervention for acute myocardial infarction. J. Thorac Cardiovasc. Surg., 93(3): 405-414, 1987.

2 BUCKBERG, G. D.; DYSON, C. W.; EMERSON, R. C. - Techniques for administering clinical cardioplegia: blood cardioplegia. In: ENGELMAN, R. M. \& LEVITSKY, S. eds. A textbook of clinical cardioplegia. Mount Kisko, N. Y., Futura Publishing Co., 1982. p. 305-316.

3 HILL, D.; KEITH, W. J.; KELLY, J. J.; SELZER, A.; ARMSTRONG, W.; POPPER, R. W.; LANGSTON, M. F.; COHN, D. - Emergency aortocoronary bypass for impeding or extending myocardial infarction. Circulation, 43(Supl. 1): 105-110, 1971.
4 KEON, W. J. - Surgery for acute myocardial infarction and cardiogenic shock. Cardiovasc. Rev. Rep., 2: 1120, 1981.

5 KILLIP III, T. \& KIMBOLL, J. T. - Treatment of myocardial infarction in a coronary care unit. $\mathrm{Am}$. J. Cardiol., 20(4): 457-464, 1967.

6 MUNDTH, E. D.; BUCKLEY, M. J.; LEINBACH, R. C.; GOLD, H. K.; DAGGET, W. M.; AUSTEN, W. G. Surgical intervention of the complications of acute myocardial ischemia. Ann. Surg., 178(3): 379-390, 1973.

7 ROSENKRANZ, E. R.; BUCKBERG, G. D.; LAKS, H.; WULDER, D. G. - Warm induction of cardioplegia with glutamate-enriched blood in cirinary patients with cardiogenic shock who are dependent on inotropic drugs and intra-aortic balloon support. J. Thorac. Cardiovasc. Surg., 80(4): 507-518, 1983.

8 SILBER, E. N. - Heart disease. 2.ed. New York, N. Y., MacMillan Publication Co., 1987. p. 1545. 
SALERNO, P. R.; ASCHE, K. A.; CUSIMANO, R. J.; LICHTENSTEIN, S. V.; SALERNO, T. A. - Resultados atuais da cirurgia coronária em pacientes de alto risco. Rev. Bras. Cir. Cardiovasc., 3(1): 50-54, 1988.

\section{Discussão}

\section{DR. SÉRGIO ALMEIDA DE OLIVEIRA \\ São Paulo, SP}

É uma honra comentar este trabalho, liderado pelo Dr. Tomás Salerno e muito bem apresentado pelo Dr. Pedro. É um tema de difícil abordagem, mas está baseado na angina instável, que, aqui, foi definida de maneira muito rigorosa, como sendo aqueles pacientes que, mesmo na terapia intensiva, sob medicação dilatadora e alguns com balão intra-aórtico, persistem nesse quadro isquêmico. Essa síndrome, como todos sabem, tem uma importância muito grande, porque está nitidamente relacionada a um infarto iminente, ou a morte súbita, e, clinicamente ela é caracterizada por 3 situaçōes; seja a angina de recente começo, que evolui para uma situação dessa instabilidade, ou de uma angina crônica antiga que, num determinado momento, se instabiliza, ou, mesmo para a chamada síndrome intermediária, ela tem vários nomes, na literatura, e a angina instável passou a ser o mais comumente utilizado. (Slide) Este slide nos dá uma idéia. Este diagrama, tirado da literatura, nos dá uma idéia muito clara de uma teoria unitária para a insuficiência coronária. Nós sabemos que uma placa de ateroma pode sofrer alteraçōes, na sua estrutura, geralmente uma hemorragia, levando à rotura do endotélio, que está em cima dessa placa e expōe o colágeno de carga positiva. Como as plaquetas são carregadas negativamente, forma-se um trombo, que pode evoluir para oclusão, a infarto, ou sofrer açôes líticas por ação de agentes endógenos e voltar a uma situação de estabilidade, ou ficar num ciclo vicioso. Assim é que essa situaçăo exige, nós sabemos, uma abordagem o mais precoce possivel. Há muitos anos, desde o início da década de 70 , nós trabalhamos para que o doente, numa situação estável, seja estudado o mais precocemente possível, discutido, e, hoje, os recursos são maiores, mais amplos, para que eles sejam tratados e não cheguem a essa situação de um grave comprometimento ventricular. $O$ que nāo ficou muito claro, talvez, na definição do risco do doente, chamado de alto risco, é que a disfunção ventricular, aqui, muito bem expressa por fração de ejeção, pode ser enganosa, especialmente nessa situação, porque o doente com a angina, nós sabemos, tem um prognóstico bom; então, a disfunção ventricular é desencadeada por um processo isquêmico e a angina é a expressão máxima disso; pode ser uma situação reversível e, freqüentemente, é reversível. Então, quando temos um doente com $20 \%$ de fração de ejeção e com dor, este significado é completamente diferente do doente que tem $20 \%$ de fração de ejeção e está em insuficiência cardiaca e não tem dor. Juntar todos esses dados num grande grupo só fica um pouco difícil. Da experiência do Dr. Salerno, ficou muito claro que, nesse grupo, primeiro maior, nenhum tinha infarto definido como tal e, do grupo em choque cardiogênico, apenas 5 tinham infarto transmural. Tratava-se, na realidade, de um grupo com muita isquemia, um grupo grave, como nós sabemos, mas que poderá ter um prognóstico muito bom. A mensagem que eu deixo é que nós não devemos aguardar, passivamente, que a situação cheque a essa gravidade. É possível que uma integração maior entre o clínico e o cirurgião, ainda mais hoje em dia, em que se dispõe de muitos recursos, com o advento dos agentes trombolíticos, por exemplo, talvez se vá poder, primeiramente, evitar chegar a uma situação de disfunção ventricular grave, pelo abortamento do quadro agudo; em segundo lugar, estabelecer, em comum acordo com o clínico, a melhor opção terapêutica. Nos Estados Unidos, durante muito tempo e, talvez, conduzidos por informaçōes da literatura, havia a idéia de que tratar um doente com angina instável era perigoso e oferecia alta incidência de infarto e alta mortalidade. Porisso, o paciente era mantido em terapia intensiva e tratado exageradamente, até com balão intra-aórtico, para, só depois, num fracasso nessa tentativa de "esfriar", é que se encaminhava esse paciente à cirurgia, o que parece uma conduta inadequada. $O$ trabalho aqui apresentado é excelente e, felizmente, conclui, acho, da mesma maneira que nós pensamos. Eu cumprimento os autores e o auditório, por ter assistido a esta excelente exposição. Obrigado.

\section{DR. MIGUEL MALUF São Paulo, SP}

Gostaria, inicialmente, de cumprimentar o Dr. Pedro Salerno e colaboradores, pela excelência do trabalho, a expressividade que sua casuística e, ao mesmo tempo, agradecer à Comissão Organizadora deste congresso, pela oportunidade que dá para discussão do assunto. O rol da revascularização do miocárdio em pacientes com insuficiência coronária, sintomáticos e que apresentam disfunção ventricular esquerda, ou seja, miocardiopatia isquêmica, não está claramente definido. Os trabalhos publicados, nesta última década, mostram resultados favoráveis, pouco favoráveis, ou contraditórios, quanto à revascularização do miocárdio, neste grupo de pacientes. Provavelmente, esta controvérsia provenha de falta de uniformidade dos critérios que definem disfunção ventricular; além disto, a seleção de pacientes com miocardiopatia isquêmica, manifestada como angina, em lugar de disfunção por fibrose miocárdica, teve um efeito dramático sobre a mortalidade cirúrgica tardia. O autor deste trabalho enfatiza, criteriosamente, a indicaçāo cirúrgica em pacientes com angina instável. A análise angiográfica da contração segmentar da parede do ventrículo esquerdo, como recomenda a classificação da American Heart Association (Circulation 51, Supl. $4: 30,1975)$, provém de critérios standard para determinar o estado funcional do ventrículo. Este método permite uma mais completa caracterização da função ventricular, mais que a fração de ejeção, ou $\mathrm{Pd}_{2}$ isoladamente, os quais são índices que refletem na função global do ventrículo. (Slide) A divisão, em 7 segmentos, do angiograma 
SALERNO, P. R.; ASCHE, K. A.; CUSIMANO, R. J.; LICHTENSTEIN, S. V.; SALERNO. T. A. - Resultados atuais da cirurgia coronária em pacientes de alto risco. Rev. Bras. Cir. Cardiovasc., 3(1): 50-54, 1988.

do ventrículo esquerdo, em posiçāo $\mathrm{OAD}$ e $\mathrm{OAE}$, e a avaliação da contração destes segmentos permitem classificar a funçăo ventricular em 5 graus, sendo que o grau 5 tem 5 , ou mais, segmentos hipo ou acinéticos; - grau 4 tem 3, ou mais, segmentos hipo ou acinéticos, e assim por diante. $\mathrm{O}$ autor usou a Canadian Classification, sendo que mais da metade dos pacientes estavam no grau III, ou IV. (Slide) Neste gráfico, observamos 2 grupos de pacientes portadores de miocardiopatia isquêmica com disfunçāo ventricular, acompanhados durante 5 anos. $O$ grupo clínico da Duke University, constituido por 1.214 pacientes, teve uma sobrevida de $89 \%$, $70 \%$ e $38 \%$, coníorme a função ventricular foi normal, moderadamente, ou difusamente comprometida, respectivamente. $\mathrm{O}$ grupo cirúrgico da University of Western Ontario, constituído por 59 pacientes, teve uma sobrevida de $86 \%$, provavelmente pela melhora da função ventricular. Este e outros trabalhos justificam a escolha do tratamento cirúrgico para este grupo de pacientes. Outro assunto que gostaria de comentar seria a proteção miocárdica, que, provavelmente, seja a chave dos resultados, neste grupo de pacientes. Nós nāo tivemos a oportunidade de pôr em prática os conceitos de Buckberg, no que se refere à proteção miocárdica e reperfusão, embora, baseado nesses trabalhos, o Dr. Ênio Buffolo tem realizado mudanças no nosso protocolo, como, por exemplo: a solução cardioplégica hiperpotássica gelada e usada na primeira injeção, dali por diante, passa a somente soro gelado sem potássio, cada 20 a 30 minutos, na raiz da aorta. Praticamos reperfusão do miocárdio, nos casos em que realizamos anastomose mamáriacoronária, ou seja, deixamos a mamária despinçada, nutrindo o miocárdio, à temparatura de $28^{\circ} \mathrm{C}$, antes de despinçar a aorta. Baixamos o fluxo da circulação extracorpórea por vários minutos, no momento de desclampear a aorta, e, nesse momento, iniciamos o aquecimento do paciente, enquanto realizamos as anastomoses proximais. Tomamos cuidado de não deixar entrar ar no sistema coronário e realizamos hiperfluxo ao fim da circulação extracorpórea, para garantir uma boa perfusão coronária. Saímos de extracorpórea sempre monitorizando as pressões de enchimento ventricular. Finalmente, a evolução dos pacientes que desenvolvem choque cardiogênico após infarte agudo do miocárdio não, necessariamente, deverá ser fatal. Cria-se a necessidade de um suporte circulatório adequado, com o uso de drogas vaso-ativas, ou mediante contrapulsaçāo com balão intra-aórtico, até definir qual é o grupo de pacientes passíveis de benefício com a revascularização do miocárdio. Este critério foi, cuidadosamente, respeitado pelo autor, desde que $40 \%$ desses pacientes apresentavam lesão do tronco de coronária esquerda. Não há lugar a dúvidas de que as mortalidades operatória e pós-operatória imediata são altas, em decorrência do estado funcional do miocárdio, ou devido às condiçōes clínicas do próprio paciente, como demonstram o trabalho aqui apresentado e outras publicaçōes. (Slide) Para estes casos, tem surgido sistema de suporte mecânico temporário dos ventrículos esquerdo e, direito, como mostra esta publicação da Universidade de Augusta, na Geórgia, onde 33 pacientes em choque caridogênico por diferentes motivos foram mantidos em circulação assisitda, até recuperar a função do ventrículo esquerdo, ou realizando um transplante cardíaco, conseguindo salvar $1 / 3$ deles. Gostaria de conhecer a experiência do Dr. Salerno, neste assunto. Como já foi sugerido por outros autores, nós achamos que a mortalidade cirúrgica, em pacientes com disfunção ventricular, depende diretamente da seleção de pacientes, onde a angina é o sintoma predominante. indicando a presença de miocárdio potencialmente viável, e da exclusão de pacientes com disfunçāo ventricular com fibrose miocárdica irreversível. Muito obrigado.

\section{DR. PEDRO SALERNO (Encerrando)}

Gostaria de agradecer os comentários feitos pelos Drs. Sérgio Oliveira e Miguel Maluf, bem como suas palavras elogiosas. Não há dúvida de que a ação do cirurgião, numa fase mais precoce do infarto do miocárdio, ou nos pacientes que estão nas condiçōes de angina instável, é necessária. A experiência da Universidade de Toronto, com a assistência ventricular esquerda, é com a Biomedical Pump. Esta bomba é constituída por um módulo de controle de fluxo ( $\mathrm{ml} /$ minuto), pressão $(\mathrm{mm} \mathrm{Hg})$, alarmes de proteção e por uma cabeça de infusão que possui, em seu interior, um imã. Através de uma cânula conectada ao átrio esquerdo, drena-se o sangue para a cabeça de infusão, que é reinfundido para a raiz da aorta, através de outra cânula. A experiência do St' Michel's Hospital da Universidade de Toronto constitui-se de 8 pacientes, nos quais o tempo de assistência ventricular variou de 12 a 24 horas. Os resultados não foram muito satisfatórios, já que 6 pacientes apresentaram assistolia, sendo, então, indicada a descontinuação da assistência ventricular $e$, nos outros 2, foi, também, descontinuada a assistência ventricular, por apresentarem parâmetros hemodinâmicos satisfatórios; porém, vieram a óbito, após 3 dias. Muito obrigado. 